\title{
Construcción y Validación de la Escala de Apreciación de la Calidad del Programa de Asignatura. Propuesta para el Estudio de la Calidad de la Docencia
}

\author{
Mauricio I. González-Arias ${ }^{(1) *}$, Edgardo Carabantes Olivares $^{(2)}$ y Nelson E. Muñoz-Carreño(1) \\ (1) Departamento de Psicología, Universidad de La Serena, Matta 147, Coquimbo-Chile \\ (email: mgonzale@userena.cl; nmunoz@userena.cl). \\ (2) Departamento de Educación, Universidad de La Serena, Amunategui 851, La Serena-Chile \\ (email: ecarabantes@userena.cl) \\ * Autor a quien debe ser dirigida la correspondencia
}

Recibido Jun. 19, 2015; Aceptado Ago. 14, 2015; Versión final Ago. 26, 2015, Publicado Feb. 2016

\begin{abstract}
Resumen
El estudio que se presenta tuvo como objetivo construir y determinar las propiedades psicométricas de una escala de apreciación de la calidad de los programas de una asignatura, por parte de los estudiantes en un curso universitario. Participaron voluntariamente 423 estudiantes de 24 carreras de una universidad regional. Respecto a la validez del constructo, el análisis de componentes principales arrojó una solución de 3 factores que explican el 55,3\% de la varianza y muestran coherencia teórica. Respecto a la validez de criterio, la correlación entre el instrumento propuesto y otro que mide un constructo relacionado fue de $r=$ 0.65 y significativa. El alfa de la escala total fue de 0.927 . Este instrumento servirá para medir la calidad de la docencia y puede ser respondido por el profesor, por un panel de expertos y por los estudiantes.
\end{abstract}

Palabras clave: calidad de la docencia, programas de asignatura, servicios educativos, construcción de test

\section{Construction and Validation of a Quality Assessment Scale in a Course Program. A Proposal to Study the Quality of Teaching}

\begin{abstract}
The objective of the study presented in this paper was to construct and to establish the psychometric properties of a scale for assessing the quality of a course program, by the students in a university course. A total of 423 students of a regional university voluntarily participated in the study. Regarding the construct validity, the analysis of the main components yielded a solution of 3 factors that explain $55.3 \%$ of the variance and show theoretical coherence. Regarding the criterion validity, the correlation between the proposed instrument and one that measures a related construct was $r=0.65$ and statistically meaningful. The alpha of the total scale was 0.927 . This instrument will helm in measuring the quality of teaching and can be answered by the teacher, by a panel of experts and by students.
\end{abstract}

Keywords: quality of teaching, course program, educational services, test construction 


\section{INTRODUCCIÓN}

Los programas de asignaturas constituyen un aspecto muy relevante y muy poco abordado, en el marco del estudio de la calidad de los procesos de formación universitaria (Nadal, 2005). Es relevante porque en el actual contexto de cambios en la educación superior, se requiere encontrar herramientas de gestión y a la vez didácticas, que permitan maximizar los esfuerzos de las instituciones de educación superior por formar profesionales no solo competentes, sino también íntegros y autónomos. El contexto de cambio se produce, entre otros aspectos, por la masificación del ingreso a la educación superior, las formas de financiamiento, los cambios tecnológicos, etc. A lo anterior hay que agregar los malos resultados de la formación previa, especialmente en la educación media, que evidencian un bajo rendimiento de los estudiantes en comprensión lectora, en matemáticas y en capacidad para resolver problemas (Agencia Calidad en la Educación, 2003). En cuanto a la docencia, las instituciones de educación superior deben hacer un esfuerzo por alcanzar el mejor resultado de formación en el menor tiempo posible, en estudiantes con un perfil de competencias de ingreso muy heterogéneo (Améstica, et al, 2014; Meléndez et al, 2010, Soria-Barreto y Zúñiga-Jara, 2014). Además, se ha producido un cambio de foco desde la enseñanza centrada en el profesor, hacia el aprendizajecentrado en el alumno (Cortés et al, 2014). Y Junto con lo anterior,se suma la necesidad, por parte de las instituciones, de acreditar la calidad de los procesos de formación de manera pública y formal (Geoffroy, 2014, Llarena et al, 2014).

Tanto a nivel institucional como al interior de las unidades a cargo de la formación de pregrado (Carreras o Programas de formación), se ha instalado el debate acerca de cómo asegurar y cómo dar cuenta de manera formal de la calidad de la docencia que se realiza. Para abordar este desafío se debe primero precisar lo que se entenderá por calidad y segundo, identificar aquellos aspectos del proceso de formación que son claves para una docencia de calidad. Respecto a la calidad existen múltiples definiciones, la Real Academia de la lengua Española (2015) define calidad como una propiedad o conjunto de propiedades inherentes a una cosa que permiten apreciarla como igual, mejor o peor que las restantes de su especie. Los procesos de acreditación definen calidad como el grado en el que un conjunto de características inherentes cumple con requisitos, que pueden ser establecidos tanto intrínseca como extrínsecamente (Geoffroy, 2014). Por su parte, la docencia es una actividad muy compleja que implica, en primer lugar, una interacción entre dos o más seres humanos, los actores, donde los aspectos subjetivos son centrales. En segundo lugar, como proceso, abarca diferentes momentos y diferentes factores que se deben conjugar. Entre los momentos se puede identificar una etapa inicial o de preparación, una etapa de implementación y una etapa de resultados. Entre los factores tenemos múltiples variables asociadas a insumos materiales y tecnológicos, económicos y de tiempo (Pereira, 2011).

Hay múltiples características de los actores que son importantes para el proceso tales como, sus conocimientos y competencias, sus motivaciones, actitudes, compromiso, hábitos, etc. Cada uno de estos aspectos puede juzgarse en su calidad de acuerdo a su aproximación a un ideal (Alcántara, 2007). Por ejemplo, ser competente es mejor que no serlo, estar motivado, es mejor que no estarlo, etc. Pero también se puede juzgar su calidad en función de su relevancia para los resultados del proceso de aprendizaje, es decir, en función de cuánto contribuyen al logro de los aprendizajes esperados para una asignatura, para el logro del perfil de egreso o para el posterior desempeño profesional. En cuanto a las etapas del proceso, el énfasis de la evaluación puede estar puesto en la planificación y ejecución de lo planificado, en los resultados, o en ambos.

La calidad se puede evaluar solamente como cumplimiento de lo propuesto, se puede evaluar a partir de los promedios de rendimiento obtenidos por los alumnos y los porcentajes de aprobación o a partir de un análisis cualitativo sobre los logros de aprendizaje. Por su parte, los demás aspectos señalados genéricamente como factores y que se refieren a los insumos materiales como la infraestructura y recursos bibliográficos, la disponibilidad de tecnología, los servicios de apoyo estudiantil, la distribución de tiempo de las actividades académicas, también cumplen un rol muy relevante en las diferentes etapas del proceso. Por ejemplo, las dimensiones y equipamientos de las salas de clases, la disponibilidad de recursos bibliográficos, etc., evaluados en función de parámetros o estándares establecidos por la misma institución o por otros organismos, también se deberían considerar en la determinación de la calidad de la docencia.

Estos aspectos permiten hacer un juicio sobre la calidad de la docencia, aunque cada uno puede tener un aporte muy distinto durante el proceso y en los resultados. La valoración de cada uno de estos aspectos se hace (y debería hacerse) a partir de las conclusiones de investigaciones científicas, sin embargo, hay que tener en cuenta que el peso relativo de cada uno y la forma en que interactúan, serán diferentes en distintas instituciones, carreras e incluso tal vez entre diferentes personas. Sin embargo, independientemente de lo anterior es posible sostener que la calidad de la docencia se puede estimar indirectamente a partir de múltiples indicadores, como por ejemplo, puntajes de ingreso de los alumnos, competencias de ingreso, 
grados académicos y perfeccionamiento específico de los profesores, tasas de aprobación, tiempos de titulación, etc., como lo tienen claro quiénes han participado en procesos de autoevaluación y acreditación (Blanco, 2007; Cortés et al, 2014; Geoffroy, 2014; Paredes, 2008;Rocha, 2013).

Sin embargo, si se considera la gran cantidad de indicadores, los costos de su registro y análisis permanente, más lo complejo de su integración e interpretación, torna poco funcional el procedimiento para operar a una escala más pequeña del proceso y para tener una mirada más integradora de los factores relevantes de la docencia focalizada en el proceso de enseñanza-aprendizaje, en una asignatura específica. Así, considerando que la mayoría de los aspectos antes mencionados confluyen y se encuentran en la organización de cada asignatura en particular, y que estos a su vez se planifican y se gestionan por medio del programa de asignatura, se puede decir que el programa puede ser considerado un foco de análisis relevante para la estimación de la calidad de la docencia (Nadal, 2005). Por ejemplo, al analizar los programas se puede observar la relación entre las asignaturas con el perfil de egreso y dentro de las asignaturas, la pertinencia de las metodologías y formas de evaluación con los objetivos de aprendizaje. Además, se puede ver la relación de los objetivos de aprendizaje (o los aprendizajes esperados) con la infraestructura que se dispone, considerando salas de clases, laboratorios, bibliotecas, etc. (Rocha, 2013; Soria-Barreto y Zúñiga-Jara, 2014).

Por lo anterior, en este artículo se propone que el estudio de los programas de asignatura puede ser una buena manera de ubicarse en un punto intermedio entre niveles y actores en el proceso de formación. Esto porque, como se ha señalado, los programas de asignaturas están en el corazón del proceso de enseñanza aprendizaje y constituyen el mapa panorámico de las actividades, tareas y criterios de aprobación que el estudiante deberá realizar para aprobar la asignatura. En ese sentido, el programa vincula la propuesta formativa de la asignatura con el estudiante. Por otro lado, vincula a la asignatura con el perfil de egreso de la carrera, especificando lo que la asignatura aporta al logro de ese perfil de egreso. Además, la mayoría de las veces, el programa de asignatura da cuenta indirectamente de las competencias del profesor y sus preferencias por determinadas didácticas y formas de evaluación y también, con los recursos y políticas institucionales, con la disponibilidad de recursos bibliográficos, laboratorios, entre otros aspectos (Nadal, 2005, Salgado et al, 2012).

\section{Calidad del proceso de formación y la satisfacción de los estudiantes en la educación superior}

Revisando diferentes aproximaciones al concepto de calidad en la educación superior Tünnermann (2000), de acuerdo con la Declaración Mundial de Educación para todos, emitida por la Conferencia de Jomtiend en 1990, señala que la calidad debe basarse no en lo que se enseña sino en lo que se aprende, poniendo así el foco en el estudiante. A su vez, la declaración Mundial de la UNESCO sobre la Educación Superior en el Siglo XXI (1998,a,b), señala que el concepto de Calidad de la Enseñanza Superior es pluridimensional y debe incluir todas las funciones y actividades que implica, tales como enseñanza, personal, edificios e instalaciones, sin embargo, al momento de evaluar la calidad, esta debe contextualizarse, por ejemplo, si una universidad recibe estudiantes académicamente desventajados, el criterio de calidad para estas instituciones debe ser el valor agregado, no el valor absoluto del producto final. Por otro lado, de acuerdo al Centro Interuniversitario de Desarrollo (CINDA; 2007) la calidad de la enseñanza universitaria, debiera observarse en aspectos tales como: la contribución al crecimiento de las economías nacionales, la integración y cohesión de las sociedades y la colaboración que puedan prestar al fortalecimiento y perfeccionamiento de las instituciones necesarias para la gobernabilidad democrática.

Por otro lado, la mayoría de los instrumentos para evaluar la calidad de la docencia impartida o la calidad de los servicios educacionales de una universidad, se construyen a partir de escalas de opinión o escalas de satisfacción. Las escalas de opinión se asemejan mucho a las escalas de actitud, sin embargo, al ser aplicadas en el contexto de la evaluación de la calidad de la docencia, suelen confundirse con escalas de satisfacción. En este sentido, la medición de la satisfacción ha cobrado relevancia al aplicar la lógica del mercado a las universidades, y se considera la satisfacción del estudiante, en cuanto usuario, como un importante indicador de la calidad de una institución. Así, la satisfacción de los usuarios de un bien o servicio puede entenderse como la relación entre las expectativas y el resultado percibido del servicio prestado (Pereira, 2011).

Por esta u otras razones, algunas universidades, a nivel institucional, han implementado sistemas de evaluación de la calidad centradas en la medición de la satisfacción de los estudiantes con la docencia recibida (Salazar, 2010), lo que resulta positivo por cuanto permite conocer la opinión de parte de uno de los actores relevantes del proceso y hacerlo de manera relativamente económica, sin embargo, se corren varios riesgos. Por ejemplo, si los instrumentos utilizados no son psicométricamente adecuados, por falta de validez se puede terminar confundiendo la medición de la satisfacción con la medición de la calidad y por falta de confiabilidad se pueden sacar conclusiones erróneas sobre el nivel de calidad estimado a partir de 
la medición. Por otro lado, si se utilizan como única medida para la evaluación de la calidad, puede distorsionar la observación realizada haciendo invisibles otros aspectos de igual o mayor relevancia para determinar la calidad del proceso. Además, puede tener el efecto indeseado de cambiar el foco de atención de los profesores hacia la satisfacción de los estudiantes exclusivamente (Paredes, 2008; Schuck et al, 2008).

Considerando lo planteado anteriormente, Torres y Araya (2010) han propuesto un instrumento para medir la calidad de los servicios educativos de las universidades chilenas a partir de la satisfacción de los estudiantes con diversos aspectos implícitos en el proceso de enseñanza-aprendizaje, tales como actitud y comportamiento del profesor, competencias del profesor, trato y servicio prestado por el personal administrativo, infraestructura y la organización de los cursos y malla curricular. En este sentido el foco de la medición es la institución en términos generales, aunque visto desde la carrera que cursa el estudiante. Como resultado obtuvieron una escala con muy buenos niveles de validez y confiabilidad. Por las características de este instrumento, en este estudio será utilizado como criterio independiente para evaluar la validez del instrumento para medir la satisfacción con los programas de asignatura que se propone.

En la misma línea, De la Fuente et al (2010) en una investigación con estudiantes de Ingeniería de la Universidad de Talca, en Chile, buscaron identificar las variables relacionadas con los niveles de satisfacción y establecer la relación entre los niveles de satisfacción y la mayor o menor fidelidad de los usuarios en el futuro. Encontraron que los aspectos que inciden mayormente en la satisfacción son la atención prestada por el personal de la Universidad, la actitud, el comportamiento y la competencia del profesor. En el otro extremo, el estudio concluye que el aspecto que no incide en la satisfacción del estudiante se relaciona con los servicios periféricos que ofrece la institución, como por ejemplo, los servicios de casino.

Aunque a nivel internacional la investigación sobre la satisfacción del usuario es de larga data, en el ámbito específico de la satisfacción de los estudiantes de Educación Superior, el interés por investigar es reciente, como lo muestran estudios realizados en diferentes partes del planeta. En China, por ejemplo, Zhanget al (2008) midieron diferentes variables asociadas a la satisfacción, con el fin de construir un índice de satisfacción de estudiantes universitarios y encontraron que una de las variables que incide en los niveles de satisfacción estudiantil, es la propia actividad desarrollada al interior de las universidades por parte de los estudiantes. Por otro lado, un estudio de la Universidad de Bari (Petruzzellis et al, 2006) muestra que la satisfacción puede estar asociada con variables como el grupo social al que se pertenece, el tipo de escuela secundaria al que pertenecían antes de ingresar a la Universidad o el desempeño en el ámbito laboral. A su vez, una investigación con estudiantes de dos universidades sudafricanas (Jager y Gbadamosi, 2010), encontró que la satisfacción estudiantil es un constructo multidimensional asociado a diversas variables, tales como: internacionalización de la Universidad reflejada en su capacidad de atraer estudiantes extranjeros, confianza en la gestión de las autoridades de la institución, intención de abandonar los estudios, calidad académica, instalaciones, etc.

La breve síntesis de estudios nacionales e internacionales presentada en los párrafos precedentes muestra, a simple vista, lo complejo que es determinar los factores claves para medir la calidad y que han predominado los estudios que evalúan la calidad a partir de la satisfacción de los estudiantes. Pero lo más importante, es que muestra que los resultados obtenidos están muy influidos por el contexto en el que se encuentra la universidad. La revisión de la literatura también ha evidenciado que no existen propuestas de instrumentos para medir la calidad a partir de los programas de asignaturas con excepción del trabajo de Nadal (2005).

\section{Medición de la calidad del programa de asignatura}

Considerando lo complejo que es medir la calidad del proceso de formación en las universidades, contar con un instrumento para evaluar el programa de asignatura puede contribuir en diferentes niveles del proceso de formación. Desde el punto de vista de los diferentes actores, puede permitir evaluar la pertinencia y ajuste del programa por el propio académico y a la vez por un grupo de pares. Esta evaluación no solo permitiría una reflexión y mejoramiento sistemático del programa en su estructura interna, sino que también en su alineamiento con los demás programas y de estos con el perfil de egreso. Suele ocurrir que al inicio de una carrera los programas están bien alineados, sin embargo, con el tiempo, los profesores van introduciendo cambios con el propósito de mejorar el programa y estos cambios van paulatinamente desalineándolos con los demás programas e incluso con el perfil de egreso. Además, este instrumento permitiría evaluar el programa desde la perspectiva del estudiante, incorporándolos así en la evaluación, lo que enriquecería la información obtenida y los resultados. Pero además, de ser aplicado sistemáticamente, permitiría a los estudiantes una reflexión más profunda sobre la utilidad del programa como guía de su propio proceso de aprendizaje. 
La propuesta hecha en el párrafo anterior es posible porque el programa de asignatura es la instancia donde confluyen la mayoría de los actores y niveles involucrados en el proceso de formación señalados previamente. En este sentido se entiende al programa de asignatura como el documento formal que contiene la presentación y organización de las materias de un curso o asignatura, los aprendizajes esperados y las formas de evaluación, entre otros contenidos. Sus formatos son variables según la institución, sin embargo, tienen una mayoría de elementos en común, como los señalados previamente (Nadal, 2005).

\section{Desarrollo de la escala de apreciación de la calidad del programa de la asignatura.}

A partir de los procesos de acreditación de la carrera de psicología de una universidad estatal regional, en Chile, uno de los autores de este artículo construyó una lista de cotejo para evaluar los programas de asignatura, de manera que todos los programas fueran visados y validados por la carrera, como una forma, entre otras, de mejorar la calidad de la docencia. En este caso, la docencia es vista como una acción colectiva más que individual en la que se requiere una mirada tanto transversal como longitudinal, al interior del plan de estudios, de los procesos de enseñanza aprendizaje que conducen al logro del perfil. Los programas fueron visados por un panel de profesores de la unidad académica, que se constituyó preferentemente de al menos dos académicos del mismo nivel en que se da la asignatura más un tercero con afinidad temática con la materia del programa.

Para realizar la evaluación, el panel utilizó la denominada pauta de validación del programa de asignatura, que tiene un formato de lista de cotejo y que sirve para constatar la presencia o ausencia de un conjunto de aspectos considerados claves en un programa de asignatura y la relación de consistencia entre ellos. Estos aspectos son: Objetivos de aprendizaje y contenidos; Aspectos metodológico-didácticos; Aspectos formales; y una apreciación general. Estos aspectos pueden considerarse también dimensiones y cada una es medida a través de un conjunto de ítems. Respecto a los Objetivos y contenidos, los ítems buscan determinar la forma en que éstos se formulan, la forma en que se relacionan con el perfil de egreso y su vínculo con otras asignaturas afines, la cantidad de objetivos y contenidos, el nivel de actualización desde la perspectiva de la disciplina y las referencias bibliográficas que los sustentan tanto en su actualización y pertinencia como en su disponibilidad en biblioteca o bases de datos. Respecto a los Aspectos metodológico-didácticos, se consideran las actividades didácticas y de evaluación en cuanto a su formulación explícita, la pertinencia con los objetivos de aprendizaje y que sean variadas (que incluya al menos más de una). Además, se considera que tenga criterios y ponderaciones explícitas y claras para las evaluaciones.

En los Aspectos formales del programa se considera una contextualización clara y completa que explicite su relación con el perfil de egreso y las competencias que aborda. Además incluye la presentación del programa en un formato estándar (de la universidad o de la carrera) y la prolijidad en la redacción, la ortografía y el orden. Finalmente, la Apreciación general considera una evaluación sobre la utilidad del programa para los alumnos en la planificación de sus tiempos para el logro de los objetivos de la asignatura y para el logro del perfil de egreso. Esta lista de chequeo fue perfeccionada y validada por los profesores del departamento de psicología y ha sido utilizada con muy buenos resultados.

A partir de esta pauta, se elaboró la Escala de Apreciación de la Calidad del Programa de Asignatura (EACPA), adaptándola para incorporar a los alumnos como fuente de información adicional a la proporcionada por el panel de profesores y así tener una visión más completa de la percepción de calidad. También, se espera que el uso del instrumento sensibilice a los estudiantes para generar en ellos un aprendizaje de cómo aprovechar el programa de asignatura como guía para rendir de manera óptima una asignatura. Para hacerlo, se transformó esta pauta, que como se dijo tenía el formato de una lista de cotejo, al formato de una escala Likert, conservando la misma formulación inicial de los ítems. Se optó por denominarla escala de apreciación y no de satisfacción porque el instrumento no pretende medir el grado en que los estudiantes están satisfechos con el programa, sino que el grado en que ellos consideran que se cumple con determinados criterios.

Así, el propósito de este artículo es proponer una escala confiable y válida para conocer la apreciación que los estudiantes hacen sobre un programa de asignatura. Los objetivos específicos son a) determinar la validez de constructo de la escala por medio de una análisis de componentes principales, b) determinar la validez referida a criterio independiente de la escala por medio del estudio de la correlación con un instrumento que mide un constructo relacionado teóricamente, c) determinar la confiabilidad por consistencia interna de la escala y d) describir la apreciación de la calidad del programa de asignatura qué hacen los estudiantes y su satisfacción con los servicios universitarios en relación con el nivel o año que cursan y el sexo. 


\section{METODOLOGÍA}

\section{Participantes}

Participaron voluntariamente en este estudio 423 estudiantes de ambos sexos, de los primeros y terceros años de 24 Asignaturas de las cuatro facultades de una Universidad Estatal Regional (Facultad de Ciencias, Facultad de Humanidades, Facultad de Ingeniería y Facultad de Ciencias Sociales y Económicas). Las carreras fueron, Ingeniería Industrial (22\%), Arquitectura (21\%), Educación Diferencial (17\%), Psicología (14\%), Químico Laboratorista, (12\%), Periodismo (8\%) y Pedagogía en Historia y Geografía (6\%). La muestra fue intencionada (no aleatoria) y se constituyó a partir de la disposición de diferentes académicos para facilitar parte del tiempo de una clase para aplicar los instrumentos.

\section{Escala de Apreciación de la Calidad del Programa (EACPA).}

Este instrumento pretende medir la calidad del programa a partir del juicio de los alumnos sobre diversos aspectos contenidos en el Programa de Asignatura, tales como: objetivos, contenidos, estrategias metodológicas- didácticas, procedimientos de evaluación, referencias bibliográficas y utilidad prestada como guía para el estudio. Está conformado por 20 ítems en formato de Escala Likert con una gradación de 1 a 5 , separados en tres dimensiones que son: Calidad de Objetivos y Contenidos, conformada por 7 ítems; Calidad Didáctica y Evaluación compuesta por 8 ítems; y Resguardo de Aspectos Formales, que considera 5 ítems. A mayor puntaje mejor es la calidad del programa. Como se señaló, esta escala se origina en una lista de cotejo diseñada para evaluar y validar programas por el profesor que dicta la asignatura y por un panel de expertos.

\section{Escala de Satisfacción Estudiantil / Calidad de los Servicios Educativos de las Universidades (U-CalS}

Este instrumento (Torres y Araya, 2010), mide la satisfacción de los estudiantes con diversos aspectos de los servicios educativos de las Universidades, específicamente: actitud y comportamiento del profesor, competencias del profesor, malla curricular, personal administrativo, instalaciones y organización de los cursos. Las respuestas se estructuran en un formato Likert con una graduación de cinco alternativas de respuestas. El Alfa de Cronbach reportado por los autores del instrumento fue de 0.88. Las dimensiones de este instrumento son Actitud y Comportamiento del Profesor, que consta de 4 ítems, Competencias del Profesor, compuesta por 3 ítems; Personal Administrativo que considera 5 ítems; Malla Curricular conformada por 5 ítems; Instalaciones con 3 ítems; y Organización de los cursos con 3 ítems. Los datos de este instrumento se utilizarán como criterio independiente para validar las puntuaciones de la EACPA.

\section{Procedimiento}

De acuerdo a la clasificación de Montero y León (2005), este estudio correspondería a un diseño Instrumental en que se busca conocer las propiedades psicométricas de la Escala de Apreciación de la Calidad del Programa de Asignatura (EACPA). Para la recolección de los datos, se eligieron dos carreras por Facultad de acuerdo a un criterio de conveniencia y de cada carrera se consideró a estudiantes que cursaran primer y tercer año con el fin de controlar la experiencia de los estudiantes en la universidad. Para acceder a los estudiantes se solicitó a los encargados de las carreras que intercedieran para contactar a docentes que dictasen asignaturas en estos cursos y que tuviesen la disposición para colaborar con la investigación. Finalmente, se conversó de manera personal con los académicos para coordinar los horarios y salas en los que se pudiese aplicar los instrumentos. Se administraron los dos instrumentos consecutivamente para medir una asignatura. Primero se aplicó el U-CalS y a continuación el EACPA. Cabe señalar que a los mismos estudiantes se les volvió a aplicar los mismos instrumentos para medir una segunda asignatura. Todas las aplicaciones fueron hechas por uno de los investigadores, quien informó previamente a los estudiantes el carácter voluntario de la participación y la confidencialidad de la información recogida, lo que se respaldó por medio de la firma de un consentimiento informado.

\section{RESULTADOS}

El objetivo de este estudio ha sido proponer una escala para medir la Apreciación de Calidad del programa de Asignatura que pueda servir como un complemento para evaluar la calidad de la docencia, como una herramienta para el mejoramiento continuo de los programas de asignaturas y su utilización como herramienta para guiar el aprendizaje por parte de los estudiantes y del profesor. Se exponen los resultados organizados de acuerdo a los objetivos específicos y en función del reporte de diferentes evidencias de la validez y la confiabilidad del instrumento propuesto. Adicionalmente, se reportan algunos resultados descriptivos sobre las puntuaciones promedios en cada escala y componentes o subescalas de los instrumentos aplicados analizados en función de variables sociodemográficas. 


\section{Validez de la Escala de Apreciación de la Calidad del Programa de Asignatura (EACPA)}

Validez por medio de análisis de componentes principales. Respecto a la validez del EACPA, luego de chequear que era posible realizar un análisis de componentes principales, a través del índice $K M O(0.927)$ y la prueba de esfericidad de Bartlett $\left(X^{2}=7007,86 ; g l=190 ; p=0.000\right)$, se procedió a analizar la validez de constructo del instrumento por medio de un análisis exploratorio. Se optó por el método de componentes principales ya que el instrumento es una lista de apreciación donde no se supone que los ítems son variables dependientes de un constructo latente (Abell et al., 2009). Además, se optó por una rotación ortogonal ya que teóricamente y a priori, los componentes no estarían correlacionados.

Al realizar el Análisis de Componentes Principales con rotación Varimax, se encontró una solución con 3 factores que en conjunto explican el $55,3 \%$ de varianza total (ver tabla 1). El primer componente se denominó "Calidad de Objetivos y contenidos", conformándose por 7 ítems que hacen referencia a objetivos y contenidos del programa de estudio. El segundo componente, al que se denominó "Calidad Didáctica y Evaluación", quedó compuesto por 6 ítems que miden, por un lado, aspectos vinculados a la didáctica como las actividades de aprendizaje propuestas por el programa, así como también miden aspectos relacionados con los procedimientos de evaluación. El tercer componente fue denominado "Cumplimiento de aspectos formales". Este componente quedó conformado por 7 ítems, que se relacionan con indicadores referidos al formato del programa, pertinencia de las referencias bibliográficas, claridad de la redacción, etc.

Tabla 1: Componentes de la escala de Apreciación de la Calidad del Programa de Asignatura

\begin{tabular}{|c|c|}
\hline Componentes determinados a partir del análisis de componentes principales & $\begin{array}{c}\text { Peso } \\
\text { factorial }\end{array}$ \\
\hline \multicolumn{2}{|l|}{ Componente 1 Calidad de Objetivos y Contenidos } \\
\hline Los objetivos se relacionan con el perfil y la naturaleza de la asignatura & 0.774 \\
\hline $\begin{array}{l}\text { La cantidad de objetivos generales y específicos es adecuada y proporcionada a la naturaleza de la } \\
\text { asignatura }\end{array}$ & 0.711 \\
\hline $\begin{array}{l}\text { Los objetivos se formulan de manera explícita y clara de acuerdo a criterios pedagógicos, como aprendizajes } \\
\text { esperados }\end{array}$ & 0.698 \\
\hline Los contenidos son pertinentes desde la perspectiva de la disciplina y/o la profesión & 0.696 \\
\hline Los contenidos están explícitos y claramente relacionados con los objetivos de aprendizaje & 0.678 \\
\hline Los contenidos están actualizados desde la perspectiva de la disciplina y/o la profesión & 0.646 \\
\hline Los contenidos/objetivos no se superponen con otros programas de la Carrera & 0.455 \\
\hline \multicolumn{2}{|l|}{ Componente 2 Calidad Didáctica y Evaluación } \\
\hline Se consideran diferentes formas de evaluación que movilicen diferentes recursos de los estudiantes & 0.755 \\
\hline $\begin{array}{l}\text { Los métodos didácticos propuestos son variados y preferentemente consideran la participación activa de los } \\
\text { estudiantes }\end{array}$ & 0.743 \\
\hline El programa explicita claramente las actividades de evaluación y estas son coherentes con los objetivos & 0.545 \\
\hline $\begin{array}{l}\text { La descripción general de la asignatura es clara, completa y explicita el área y/o las competencias que } \\
\text { aborda }\end{array}$ & 0.523 \\
\hline El programa explicita claramente las actividades didácticas y estas son coherentes con los objetivos & 0.516 \\
\hline El programa permite visualizar claramente su relación con el perfil de egreso & 0.494 \\
\hline \multicolumn{2}{|l|}{ Componente 3 Resguardo de Aspectos Formales } \\
\hline Al menos parte de las referencias básicas se encuentran en Biblioteca & 0.703 \\
\hline $\begin{array}{l}\text { Se adjunta una calendarización del avance de las actividades y de las evaluaciones } \\
\text { durante el semestre }\end{array}$ & 0.601 \\
\hline Las referencias bibliográficas son pertinentes y actualizadas & 0.561 \\
\hline El programa está presentado respetando el formato de la Universidad & 0.546 \\
\hline $\begin{array}{l}\text { El programa sirve al estudiante como una guía para organizar sus actividades de } \\
\text { lectura y preparación de trabajos con el fin de aprobar satisfactoriamente la asignatura }\end{array}$ & 0.509 \\
\hline El programa está redactado prolijamente, es ordenado y no tiene faltas de ortografía & 0.490 \\
\hline Las ponderaciones y criterios de evaluación son claros y explícitos & 0.470 \\
\hline
\end{tabular}




\section{Validez referida a un criterio independiente}

La correlación por medio del coeficiente rho de Spearman entre la EACPA y la U-CalS fue de $\rho=0,618$ $(p<0,001)$. Al analizar las correlaciones entre los componentes de ambos instrumentos (Tabla 2$)$, se puede ver que las correlaciones más altas se dieron entre el componente Competencias del Profesor, del instrumento que midió la satisfacción con la calidad de los servicios educativos y el componente Calidad de Objetivos y Contenidos del Instrumento que midió la apreciación de la calidad del programa de asignatura, teniendo un valor de $\rho=0,556, p<0,001$ y entre Competencias del Profesor y el componente Calidad Didáctica y Evaluación, que fue de $\rho=0,535, p<0,001$. La calidad de atención de administrativos, presentó una correlación de fuerza media con los tres componentes de apreciación de la calidad del programa. El componente Calidad del Plan de Estudios del Instrumento de Satisfacción con la Calidad de los Servicios Educativos, muestra una mayor relación con Calidad de Objetivos y Contenidos correspondiente al instrumento que midió la Apreciación de la calidad del programa de asignatura, presentando un valor de $\rho=$ $0,469, p<0,001$. A su vez la calidad de la infraestructura presenta la correlación más baja con el componente Calidad didáctica y evaluación, manifestado en un valor de $\rho=0,251, p<0,001$. El componente Organización del Curso exhibe también una más fuerte correlación con el componente Calidad de Objetivos y Contenidos, con un valor de $\rho=0,437, p<0,001$ (Ver Tabla 2).

Tabla 2: Correlaciones (Rho de Spearman) entre los Componentes de la Escala de Apreciación de la calidad del Programa de Asignatura y la Escala de Satisfacción con la calidad del Servicio Educativo en Universidades.

\begin{tabular}{|l|c|c|c|c|c|c|c|c|}
\hline Componentes & 1 & 2 & 3 & 4 & 5 & $a$ & $b$ & $c$ \\
\hline $\begin{array}{l}\text { 1.- Competencia del } \\
\text { Profesor }\end{array}$ & --- & $.252^{* *}$ & $.325^{* *}$ & $.145^{* *}$ & $.252^{* *}$ & $.556^{* *}$ & $.535^{* *}$ & $.471^{* *}$ \\
\hline $\begin{array}{l}\text { 2.- Calidad Atención } \\
\text { Administrativos }\end{array}$ & & & $.444^{* *}$ & $.333^{* *}$ & $.350^{* *}$ & $.379^{* *}$ & $.380^{* *}$ & $.387^{* *}$ \\
\hline $\begin{array}{l}\text { 3.- Calidad del Plan de } \\
\text { Estudio }\end{array}$ & & & & $.441^{* *}$ & $.479^{* *}$ & $.469^{* *}$ & $.424^{* *}$ & $.415^{* *}$ \\
\hline $\begin{array}{l}\text { 4.- Calidad } \\
\text { infraestructura }\end{array}$ & & & & & $.512^{* *}$ & $.341^{* *}$ & $.251^{* *}$ & $.359^{* *}$ \\
\hline $\begin{array}{l}\text { 5.- Organización del } \\
\text { Curso }\end{array}$ & & & & & & $.437^{* *}$ & $.387^{* *}$ & $.363^{* *}$ \\
\hline $\begin{array}{l}\text { a.- Calidad objetivos y } \\
\text { contenidos }\end{array}$ & & & & & & & $.705^{* *}$ & $.678^{* *}$ \\
\hline $\begin{array}{l}\text { b.- Calidad Didáctica y } \\
\text { Evaluación }\end{array}$ & & & & & & & & $.732^{* *}$ \\
\hline $\begin{array}{l}\text { C.- Cumplimiento } \\
\text { Aspectos Formales }\end{array}$ & & & & & & & &.--- \\
\hline
\end{tabular}

\section{Confiabilidad de la Escala de Apreciación de la Calidad del Programa de Asignatura}

La consistencia interna de la escala, a través del Alfa de Cronbach, fue de 0,927 y el Error Estándar de Medida (EEM; Gempp, 2006) para la prueba con 20 ítems y con una muestra de 746 casos fue de 0,16 (recordar que los estudiantes respondieron para dos asignaturas ambos instrumentos, aunque algunos solo lo hicieron para una sola). Los niveles de confiabilidad de la escala total y de cada componente del EACPA se reportan en la Tabla 3.

Tabla 3: Confiabilidad y Error Estándar de la Medición (Alfa de Cronbach) total y por componentes de la Escala de Apreciación de la Calidad del Programa de Asignatura. ${ }^{*}$ EEM = Error Estándar de Medida

\begin{tabular}{|l|c|c|c|c|}
\hline Componentes de la escala & $M$ & $D E$ & Alfa & EEM \\
\hline Calidad del Programa & 4,1 & 0,60 & 0,927 & 0,16 \\
\hline Calidad de Objetivos y Contenidos & 4,2 & 0,63 & 0,861 & 0,23 \\
\hline Calidad Didáctica y Evaluación & 4,1 & 0,71 & 0,834 & 0,29 \\
\hline Cumplimiento de Aspectos Formales & 4,1 & 0,67 & 0,817 & 0,29 \\
\hline
\end{tabular}




\section{Apreciación de la calidad del Programa de Asignatura de acuerdo al nivel y al sexo}

Los promedios están expresados en una escala de 1 a 5 para hacer más fácil su interpretación con independencia del número de ítems. El promedio en la evaluación de la calidad de los programas que se obtiene es de $M=4,1(D E=0,60)$. Al comparar la Apreciación de los alumnos de diferentes niveles se observa un incremento significativo en tercer año, tanto en la escala general como en cada una de las subescalas de la prueba tal como se puede observar en la tabla 4. En cuanto al sexo, se observa una tendencia sistemática en que los hombres reportan una mayor apreciación de calidad que las mujeres, de manera significativa, tal como se muestra en la tabla 5 . En las tablas 4 y 5 se agrega una columna con la probabilidad a partir de una prueba no paramétrica, considerando que la distribución de los puntajes no se ajusta exactamente a una distribución normal.

Tabla 4: Descripción de la Apreciación de la Calidad del Programa de Asignatura para la escala general y las sub-escalas diferenciadas por nivel en la carrera (K-W = Kruskal-Wallis)

\begin{tabular}{|l|c|c|c|c|c|c|c|c|c|}
\hline Sub Escalas & Nivel & $N$ & Media & $\begin{array}{c}\text { Desviación } \\
\text { típica }\end{array}$ & Mín. & Máx. & $\begin{array}{c}F g l \\
(1,744)\end{array}$ & $\begin{array}{c}\text { Sig. } \\
\text { ANOVA }\end{array}$ & Sig. K-W \\
\hline $\begin{array}{l}\text { Calidad del } \\
\text { Programa en } \\
\text { General }\end{array}$ & $1^{\circ}$ año & 519 & 4,062 & 0,5912 & 1,4 & 5,0 & 14,030 & 0,000 & 0,000 \\
\cline { 2 - 10 } & $3^{\circ}$ año & 227 & 4,240 & 0,6146 & 1,0 & 5,0 & & & \\
\hline $\begin{array}{l}\text { Calidad } \\
\text { Objetivos y } \\
\text { Contenidos }\end{array}$ & $1^{\circ}$ año & 519 & 4,132 & 0,6297 & 1,4 & 5,0 & 5,825 & 0,016 & 0,008 \\
\hline & $3^{\circ}$ año & 227 & 4,253 & 0,6379 & 1,0 & 5,0 & & & \\
\hline $\begin{array}{l}\text { Calidad } \\
\text { Didáctica y } \\
\text { Evaluación }\end{array}$ & $1^{\circ}$ año & 519 & 4,024 & 0,7015 & 0,5 & 5,0 & 13,282 & 0,000 & 0,000 \\
\hline $\begin{array}{l}\text { Cumplimiento } \\
\text { de Aspectos } \\
\text { Formales }\end{array}$ & $1^{\circ}$ año & 227 & 4,228 & 0,7144 & 1,0 & 5,0 & & & \\
\cline { 2 - 10 } & $3^{\circ}$ año & 227 & 4,239 & 0,6556 & 1,0 & 5,0 & & & 0,000 \\
\hline
\end{tabular}

Tabla 5: Descripción de Apreciación de la calidad del Programa de Asignatura para la escala general y las subescalas diferenciados por sexo (K-W = Kruskal-Wallis)

\begin{tabular}{|l|c|c|c|c|c|c|c|c|c|}
\hline \multicolumn{1}{|c|}{ Escala } & Sexo & $N$ & Media & $\begin{array}{c}\text { Desviación } \\
\text { típica }\end{array}$ & Mínimo & Máximo & $\begin{array}{c}F g l \\
(1,744)\end{array}$ & $\begin{array}{c}\text { Sig. } \\
\text { ANOVA }\end{array}$ & Sig. K-W \\
\hline $\begin{array}{l}\text { Calidad del } \\
\text { programa en } \\
\text { general }\end{array}$ & mujer & 277 & 4,01 & 0,6345 & 1,0 & 5,0 & 15,082 & 0,000 & 0,000 \\
\cline { 2 - 10 } & hombre & 453 & 4,18 & 0,5737 & 1,6 & 5,0 & & & \\
\hline $\begin{array}{l}\text { Calidad } \\
\text { Objetivos y } \\
\text { Contenidos }\end{array}$ & mujer & 277 & 4,064 & 0,6828 & 1,0 & 5,0 & 12,518 & 0,000 & 0,001 \\
\cline { 2 - 11 } $\begin{array}{l}\text { Calidad } \\
\text { Didáctica y } \\
\text { Evaluación }\end{array}$ & hombre & 453 & 4,233 & 0,5877 & 2,0 & 5,0 & & & \\
\hline & hujer & 277 & 3,947 & 0,7261 & 0,5 & 5,0 & 16,860 & 0,000 & 0,000 \\
\hline $\begin{array}{l}\text { Cumplimiento } \\
\text { Aspectos } \\
\text { Formales }\end{array}$ & mujer & 277 & 4,004 & 0,7062 & 0,9 & 5,0 & 7,679 & 0,006 & 0,008 \\
\cline { 2 - 11 } & hombre & 453 & 4,145 & 0,6457 & 1,4 & 5,0 & & & \\
\hline
\end{tabular}

\section{Satisfacción de los estudiantes con la calidad del Servicio Educativo Universitario}

La Satisfacción con el Servicio Educativo Universitario tuvo un promedio de $M=3,9(D E=0,54)$. Al comparar por niveles sólo se encontró una diferencia significativa en la percepción de la calidad del Plan de Estudio que fue mayor en los estudiantes de tercer año, lo que se puede ver en la tabla 6 . En cuanto al sexo, sólo se encontró que los hombres tienen una mejor percepción de la competencia de los profesores y de la calidad del plan de estudio que las mujeres, lo que se puede apreciar con más detalle en la tabla 7. Igual que en las tablas 5 y 6 , en las tablas 6 y 7 se agrega una columna con los valores de significación de una prueba no paramétrica, ya que la distribución de puntajes de las escalas no se ajustan exactamente a distribución normal. Al respecto, solo llama la atención que en esta última columna, en la tabla 7 con la prueba no paramétrica las mujeres presentan una mejor percepción de la calidad de la infraestructura que los hombres. 
Tabla 6: Descripción de la Satisfacción de los Estudiantes con la Calidad del Servicio Educativo Universitario para la escala general y las sub-escalas diferenciados por nivel ( $\mathrm{K}-\mathrm{W}=\mathrm{K}$ ruskal-Wallis)

\begin{tabular}{|c|c|c|c|c|c|c|c|c|c|}
\hline Escala & Nivel & $\mathrm{N}$ & Media & $\begin{array}{c}\text { Desviación } \\
\text { típica }\end{array}$ & Mínimo & Máximo & $\begin{array}{c}\mathrm{Fgl} \\
(1,744)\end{array}$ & $\begin{array}{c}\text { Sig. } \\
\text { ANOVA }\end{array}$ & Sig. $K-W$ \\
\hline \multirow{2}{*}{$\begin{array}{l}\text { Satisfacción } \\
\text { Calidad } \\
\text { Enseñanza }\end{array}$} & $1^{\circ}$ año & 519 & 3,907 & 0,5426 & 2,2 & 5,0 & 2,689 & 0,101 & 0,074 \\
\hline & 3ำ año & 227 & 3,836 & 0,5515 & 1,6 & 5,0 & & & \\
\hline \multirow{2}{*}{$\begin{array}{l}\text { Competencia } \\
\text { del Profesor }\end{array}$} & $1^{0}$ año & 519 & 4,401 & 0,5746 & 2,1 & 5,0 & 1,658 & 0,198 & 0,034 \\
\hline & $3^{\circ}$ año & 227 & 4,463 & 0,6465 & 1,0 & 5,0 & & & \\
\hline \multirow{2}{*}{$\begin{array}{l}\text { Calidad } \\
\text { Atención } \\
\text { Administrativos }\end{array}$} & $1^{\circ}$ año & 519 & 3,827 & 0,7716 & 1,0 & 5,0 & 1,618 & 0,204 & 0,351 \\
\hline & $3^{\circ}$ año & 227 & 3,745 & 0,8806 & 1,0 & 5,0 & & & \\
\hline \multirow{2}{*}{$\begin{array}{l}\text { Calidad del } \\
\text { Plan de } \\
\text { Estudio }\end{array}$} & $1^{\circ}$ año & 519 & 3,869 & 0,7186 & 1,0 & 5,0 & 22,824 & 0,000 & 0,000 \\
\hline & 3ำ año & 227 & 3,581 & 0,8360 & 1,4 & 5,0 & & & \\
\hline \multirow{2}{*}{$\begin{array}{l}\text { Calidad } \\
\text { Infraestructura }\end{array}$} & $1^{\circ}$ año & 519 & 3,534 & 0,9153 & 1,0 & 5,0 & 0,056 & 0,813 & 0,385 \\
\hline & $3^{\circ}$ año & 227 & 3,517 & 0,8273 & 1,3 & 5,0 & & & \\
\hline \multirow{2}{*}{$\begin{array}{l}\text { Organización } \\
\text { del Curso }\end{array}$} & $1^{\circ}$ año & 519 & 3,906 & 0,8857 & 1,0 & 5,0 & 0,198 & 0,656 & 0,471 \\
\hline & $3^{\circ}$ año & 227 & 3,874 & 0,8639 & 1,0 & 5,0 & & & \\
\hline
\end{tabular}

Tabla 7: Descripción de la Satisfacción de los Estudiantes con la Calidad del Servicio Educativo Universitario para la escala general y las sub-escalas diferenciados por nivel (K-W = Kruskal-Wallis)

\begin{tabular}{|l|l|c|c|c|c|c|c|c|c|}
\hline \multicolumn{1}{|c|}{ Escala } & Sexo & $\mathrm{N}$ & Media & $\begin{array}{c}\text { Desviación } \\
\text { típica }\end{array}$ & Mínimo & Máximo & $\mathrm{F}$ & $\begin{array}{c}\text { Sig. } \\
\text { ANOVA }\end{array}$ & Sig. K-W \\
\hline $\begin{array}{l}\text { Satisfacción } \\
\text { Calidad } \\
\text { Enseñanza }\end{array}$ & mujer & 277 & 3,848 & 0,5624 & 1,6 & 5,0 & 1,723 & 0,190 & 0,334 \\
\hline & hombre & 453 & 3,903 & 0,5324 & 2,1 & 5,0 & & & \\
\hline $\begin{array}{l}\text { Competencia } \\
\text { del Profesor }\end{array}$ & mujer & 277 & 4,345 & 0,6091 & 1,0 & 5,0 & 6,851 & 0,009 & 0.001 \\
\hline & hombre & 453 & 4,465 & 0,5895 & 1,1 & 5,0 & & & \\
\hline $\begin{array}{l}\text { Calidad } \\
\text { Atención } \\
\text { Administrativos }\end{array}$ & mujer & 277 & 3,771 & 0,8165 & 1,0 & 5,0 & 0,336 & 0,563 & 0,656 \\
\hline & hombre & 453 & 3,807 & 0,7956 & 1,0 & 5,0 & & & \\
\hline $\begin{array}{l}\text { Calidad del } \\
\text { Plan de } \\
\text { Estudio }\end{array}$ & mujer & 277 & 3,688 & 0,8038 & 1,0 & 5,0 & 6,635 & 0,010 & 0,036 \\
\hline & hombre & 453 & 3,838 & 0,7368 & 1,4 & 5,0 & & & \\
\hline $\begin{array}{l}\text { Calidad } \\
\text { Infraestructura }\end{array}$ & mujer & 277 & 3,593 & 0,9228 & 1,0 & 5,0 & 2,692 & 0,101 & 0,047 \\
\hline & hombre & 453 & 3,481 & 0,8686 & 1,0 & 5,0 & & & \\
\hline $\begin{array}{l}\text { Organización } \\
\text { del Curso }\end{array}$ & mujer & 277 & 3,845 & 0,8874 & 1,0 & 5,0 & 1,389 & 0,239 & 0,256 \\
\cline { 2 - 11 }$y$ & hombre & 453 & 3,924 & 0,8751 & 1,0 & 5,0 & & & \\
\hline
\end{tabular}

\section{DISCUSIÓN}

El objetivo de este estudio ha sido determinar las propiedades psicométricas de un nuevo instrumento denominado Escala Apreciación de la calidad del Programa de Asignatura (EACPA) que se propone como un complemento para estimar la calidad de la docencia. Este instrumento puede ser utilizado por el profesor para evaluar su programa, puede ser utilizado por una comisión de expertos para validar el programa del profesor y en lo que respecta a este estudio, se propone para recoger la apreciación de los alumnos sobre la calidad del programa y así complementar una evaluación con participación de los actores claves del proceso educativo.

En cuanto a la validez del instrumento, como se señaló en la introducción, la validez de contenido ya había sido considerada en la elaboración inicial como pauta de validación de programas incluyendo todos los elementos considerados relevantes para la validación del programa en el marco de su ajuste al perfil de egreso y su coherencia interna entre objetivos de aprendizaje, métodos didácticos, estrategias de evaluación, disponibilidad de libros en biblioteca, etc., con el fin no sólo de ir mejorando progresivamente la 
calidad técnica del programa, sino de evitar el desacople con los demás programas a lo largo de cambios sucesivos. Esta validez se obtuvo por medio de jueces expertos y ha demostrado ser muy útil en sus aplicaciones previas. En cuanto a los resultados de este estudio, los componentes extraídos por medio del análisis de componentes principales explican una buena porción de varianza y tienen alta consistencia lógica. La solución con tres componentes es parsimoniosa y clara, y está en concordancia con la estructura conceptual desde donde se origina el instrumento. El instrumento inicial contemplaba componentes denominados: Objetivos de Aprendizaje y Contenidos, Aspectos Metodológicos y Didácticos, Aspectos Formales y una Evaluación General, que finalmente quedó asimilada al penúltimo componente de Aspectos Formales. En cuanto al método de componentes utilizado, este instrumento, al ser una escala de apreciación, los ítems no pretenden ser variables dependientes de una variable latente, sino más bien, se ha pretendido agrupar los ítems en componentes para reducir la complejidad explicativa.

A su vez, la validez referida a criterio independiente, obtenida al correlacionar la Escala de Apreciación de la calidad del Programa de Asignatura con la Escala de Satisfacción con la Calidad del Servicio Educacional, demostró una correlación significativa, positiva y de fuerza media entre ambos instrumentos. Aún más, todas las sub-escalas de ambos instrumentos presentaron correlaciones significativas, pero las sub-escalas que mejor correlacionaron fueron las de Competencia del Profesor con la Calidad de Contenidos y Objetivos, con Calidad Didáctica y Evaluación y Cumplimiento de Aspectos Formales, lo que tiene consistencia lógica. En otras palabras, podría decirse que hay una relación en que altas puntaciones asignadas a los objetivos, contenidos, didáctica, evaluación y aspectos formales del programa, se corresponden con altas puntuaciones en la satisfacción con la Competencia del Profesor. Esto es muy relevante por cuanto la competencia del profesor percibida se relaciona con la calidad de los objetivos y contenidos del programa y es el aspecto que tiene una correlación más fuerte que los demás aspectos evaluados. Sería muy interesante profundizar en este aspecto en estudios posteriores. En general, y en el marco de este estudio, la evidencia obtenida permite sostener la validez de criterio del instrumento.

En cuanto a la confiabilidad, los índices de consistencia interna encontrados son los adecuados para un instrumento diseñado para realizar mediciones colectivas, según lo planteado por Aiken (1996). El margen de error del instrumento es pequeño. Este margen de error es más pequeño para la escala general y un poco más grande aunque siempre dentro de un estándar alto de adecuación, para las sub-escalas. Estos resultados muestran que el instrumento propuesto (EACPA) se comporta de manera consistente y válida al ser respondido por estudiantes, siendo un valioso aporte para validar la calidad de los programas de asignatura. Además, esto permitiría contar con un indicador adicional de la calidad de la docencia. No obstante lo anterior, se requieren de más estudios para evaluar su estabilidad y su utilidad.

Al respecto y como limitación, cabe señalar que haría falta complementar la confiabilidad del instrumento por un indicador de estabilidad, que no pudo realizarse en el marco de este estudio. En cuanto a las evidencias de validez esta podría continuar estudiándose a partir de otros instrumentos, especialmente el instrumento que se aplica para evaluar la satisfacción con la docencia cada semestre, por parte de los estudiantes en las diferentes universidades. Una vez que se tiene evidencia inicial del buen comportamiento psicométrico del instrumento, y como una forma de establecer su utilidad, se reporta un análisis a partir de los datos descriptivos de ambos instrumentos aplicados respecto a las variables nivel de estudio y sexo.

En general, se puede interpretar que la evaluación que hacen los estudiantes de los programas como de la satisfacción con el servicio prestado es buena, considerando que el valor promedio se aproxima a cuatro en una escala que tiene teóricamente promedio tres y un puntaje máximo de cinco. Sin embargo, sería interesante calibrar mejor las puntuaciones para interpretar mejor este resultado ya que en general las escalas Likert de satisfacción suelen tener medias por sobre la media teórica. Se puede observar también que ambos instrumentos son complementarios en cuanto a los ámbitos abordados, pero las correlaciones y las similitudes en los resultados permiten suponer que podría haber una variable latente asociada con la apreciación de calidad o bien de satisfacción general, que esté a la base de las puntuaciones en cada una de las escalas de ambos instrumentos. Estudios posteriores podrían abordar este desafío.

En cuanto a la Apreciación de la calidad de los Programas de Asignatura en relación al nivel que se cursa y el sexo de los y las estudiantes, en todos los componentes hay un mejor nivel de satisfacción en los estudiantes de tercer año, lo que se podría deber a que la calidad de los programas aumenta hacia tercer año o bien que las competencias de los estudiantes para evaluar los programas mejoran, o ambas. En el primer caso habría que evaluar si los profesores que hacen clases en tercer año tienen mejor calificación que los profesores que hacen clases en primero, en el segundo caso, habría que averiguar si el incremento de la formación y competencias del estudiante los hace valorar mejor las competencias de los profesores. Sin embargo, lo que habría que rescatar es que la calidad de los programas se puede incrementar si se elaboran de acuerdo a estándares rigurosos y si se validan de manera explícita por expertos. Respecto al análisis por sexo, al igual que en el instrumento U-CalS, las mujeres evalúan sistemáticamente más bajo que los hombres la calidad de los Programas de Asignatura, con la excepción de la percepción de calidad 
de la infraestructura, que resultó significativa solo en el análisis no paramétrico. Este aspecto de evaluación diferenciada relacionado al género es un tema de análisis muy interesante pero que escapa a los fines de este estudio.

Por otro lado, De la Fuente et al. (2010) plantean que aún cuando el aspecto que más incide en la satisfacción de los estudiantes tiene relación con las competencias del profesor, existen otros factores adicionales que permiten incrementar los niveles de satisfacción estudiantil, tales como la calidad de las instalaciones, el tipo de atención que brinda el personal administrativo, etc. En este caso la percepción de los estudiantes está por sobre el promedio pero sistemáticamente por debajo de la satisfacción con los profesores. No obstante, respecto a los buenos resultados psicométricos, hay que considerar que cada estudiante evaluó dos asignaturas y dos programas, lo que podría haber incrementado los valores de consistencia y las correlaciones encontradas, sin embargo, de haber ocurrido esto se espera que el efecto haya sido marginal. Como corresponde a la investigación científica sería conveniente replicar estos análisis en nuevas investigaciones.

En otro ámbito, se pueden criticar las competencias de los estudiantes para evaluar un programa de asignatura, sin embargo, si bien pueden haber estudiantes poco competentes al inicio de la carrera, gradualmente ellos se van familiarizando con los programas de estudio y se van haciendo más competentes en su manejo. Además, son los estudiantes quienes tienen el conocimiento en primera persona de las consecuencias de un programa en su proceso de formación y lo que se espera es que puedan sensibilizarse para aprender a sacarle el mayor provecho posible al programa como marco orientador y a la vez como una especie de "hoja de ruta" frente al desafío de la asignatura, en parte como han sugerido Martínez-Gómez y Marín-García (2009).

De todas maneras, hay que considerar lo complejo que es evaluar la calidad de la docencia y de la contribución relativa de los diferentes factores en la calidad vista como resultado. El impacto en el resultado del proceso de formación no obedece a una sumatoria lineal de efectos, sino a un proceso sistémico mucho más complejo que aún no se conoce del todo. En general, desde un punto de vista lógico y técnico, la mejor evaluación sería una que abordara la mayor cantidad de niveles y actores posibles, pero esto tiene costos altos. Por lo anterior, como se ha señalado, la evaluación del programa puede ser una alternativa parsimoniosa.

\section{CONCLUSIONES}

En conclusión, los resultados permiten señalar que se cuenta con un instrumento confiable y válido de manera preliminar, para abordar el estudio de la calidad de los programas de asignaturas como un elemento clave en la calidad de la docencia universitaria y en la percepción de la calidad de la docencia por los estudiantes. Adicionalmente, se puede señalar que el aspecto mejor evaluado por los estudiantes es la competencia del profesor y que este aspecto presenta la correlación más fuerte con los objetivos y contenidos del programa. Otro aspecto relevante es que las mujeres tienden a evaluar, en la mayoría de las dimensiones, más bajo que los hombres, que la apreciación de la calidad del programa se incrementa en tercer año y que en algunas dimensiones, la satisfacción con los servicios educativos disminuye.

\section{REFERENCIAS}

Abell, N., Springer D. y Kamata, A. Developing and Validating Rapid Assessment Instruments, 3-222, Oxford University Press, NY, EEUU., (2009)

Acosta, A., El Neointervencionismo Estatal en la Educación Superior en América Latina,http://www.revistasociologica.com.mx/pdf/4903.pdf, Sociológica, 17 (49), 43-72,(2002)

Agencia Calidad en la Educación, Gobierno de Chile, PISA, Programa nacional de evaluación de estudiantes, http://www.agenciaeducacion.cl/wp-content/uploads/2013/04/PISA-Programa-Internacional-deEvaluaci\%C3\%B3n-de-Estudiantes.pdf,(2003)

Aiken, L.R., Test Psicológicos y Evaluación,Prentice Hall, Ciudad de México, México, (1996)

Alcántara, A., Dimensiones de la calidad en la educación superior,http://www.redalyc.org/articulo.oa?id=34005004,ISSN: 0188-168X, Reencuentro, 50, 21-27, (2007)

Améstica, L. R, X. Llinas-Audet y I. R. Sánchez, Retorno de la Educación Superior en Chile: Efecto en la movilidad social a través del estimador de Diferencias en Diferencias, http://dx.doi.org/10.4067/S071850062014000300004, Formación universitaria, 7(3), 23-32, (2014)

Blanco, M., Las Conductas que mejor Predicen la Calidad de la Docencia según los Estudiantes 
Universitarios,http://www.scielo.org.bo/scielo.php?script=sci_arttext\&pid=S2077-

21612007000200002\&lang=pt, ISSN 2077-2161, RAP [online], vol.5, n.2, pp. 24-36, (2007)

Centro Interuniversitario de Desarrollo, CINDA. Evaluación del desempeño docente y la calidad de la docencia universitaria.http://www.cinda.cl/download/libros/EVALUACl\%C3\%93N\%20DEL\% 20DESEMPE\%C3\%910\%20DOCEN.pdf, CINDA, Santiago de Chile, Chile, (2007)

Cortés, E., M. Campos y M. P. Moreno, Priorización de las Dimensiones de Evaluación al Desempeño Docente por el Estudiante, en tres Áreas del Conocimiento, http://dx.doi.org/10.4067/S071850062014000200002, Formación universitaria, 7(2), 3-10, (2014)

De la Fuente, H.,M. Marzo y M. J.Reyes, Análisis de la satisfacción universitaria en la Facultad de Ingeniería de la Universidad de Talca,http://dx.doi.org/10.4067/S0718-33052010000300009, ISSN 0718-3305, Ingeniare, Revista chilena de Ingeniería, 18 (3), 350-363, (2010)

Gempp, R., El error estándar de medida y la puntuación verdadera de los test psicológicos. Algunas recomendaciones prácticas. http://dx.doi.org/10.4067/S0718-48082007000100004, ISSN 0718-4808, Terapia Psicológica, 24 (2), 117 - 129.(2006)

Geoffroy, E., Origen y Característica del Sistema de Aseguramiento de la Calidad de la Educación Superior Chileno. DOI: http://dx.doi.org/10.6018/reifop.17.3.204051. Revista electrónica Interuniversitaria de Formación del Profesorado, 17 (3), 49-64. (2014)

Jager, J. y G. Gbadamosi, Specific remedy for specific problem: Measuring service quality in South African higher education,DOI 10.1007/s10734-009-9298-6, High Education, 60 (3), 251-267, (2010)

Llarena, M. G., S.L. Villodre, , F. A. Pontoriero y A.B. Cattapan, Modelo de Sistema de Gestión de Calidad Para la Puesta en Marcha de Cursos no Presenciales: Instrumentos de Seguimiento $Y$ evaluación, Http://Dx.Doi.Org/10.4067/S0718-50062014000600002, Formación universitaria, 7(6), 3-16, (2014)

Martínez-Gómez, M. y Marín-García, J. A. Cómo Medir y Guiar Cambios hacia Entornos Educativos más Motivadores. doi: 10.4067/S0718-50062009000400002. Form. Univ., v 2(4) 3-14 (2009)

Meléndez, M.,P. Solís, y J. Gómez, Gobernanza y Gestión de la Universidad Pública, Revista de Ciencias Sociales, XVI (2), 210-225. (2010)

Montero, I., y O. León, Sistema de clasificación del método en los informes de investigación en Psicología,www.redalyc.org/articulo.oa?id=33701007, ISSN: 1697-2600, International Journal of Clinical and Health Psychology, 5 (1), 115-127, (2005)

Moreno, M. P., M.C. Nava y M. Campos, Los Comentarios Abiertos como Referente de Evaluación de la Docencia Universitaria: La Conveniencia de su Interpretación y Tratamiento, http://dx.doi.org/10.4067/S0718-50062014000100006, Formación Universitaria. , vol.7, n.1, 41-48. (2014)

Nadal, A. El programa universitario como herramienta de evaluación. Tesis doctoral. http://hdl.handle.net/10803/9392, Departamento de Pedagogías y Didácticas Específicas de la Universidad de las Islas Baleares, España, (2005)

Paredes, B.E., Acreditación Universitaria: Garantía de Calidad en la Educación Superior?http://www.scielo.org.pe/scielo.php?script=sci_arttext\&pid=S1018-130X2008000200001, ISSN 1729-214X, Rev Med Hered [online]. vol.19, n.2, 42-43. (2008)

Pereira, M., Nuevas tendencias en la evaluación de la calidad de las universidades: los índices de calidad percibida y satisfacción de los egresados (con modelos de ecuaciones estructurales), http://dialnet.unirioja.es/servlet/articulo?codigo=3691511, ISSN: 0210-2773, Aula Abierta 2011, Vol. 39, núm. 3, 73-84 (2011)

Petruzzellis, L.,M. D'Uggento y S. Romanazzi, Student satisfaction and quality of service in italian universities, http://www.emeraldinsight.com/doi/abs/10.1108/09604520610675694, ISSN: 0960-4529, Managing Service Quality, 16 (4), 349-364, (2006)

RAE, Real Academia de la Lengua Española (en línea), 2012. http://lema.rae.es/drae/?val=calidad, Acceso 15 de marzo de (2015)

Rocha, R., Escala de Opinión de los Estudiantes sobre la Efectividad de la Docencia (eoeed) en Educación Superior.http://dx.doi.org/10.4067/S0718-50062013000600003, Formación universitaria, 6(6), 13-22, (2013) 
Salazar, J., Encuesta de satisfacción estudiantil versus cultura evaluativa de la docencia. https://repositorio.uam.es/handle/10486/661588,ISSN: 1989-0397,Revista Iberoamericana de Evaluación Educativa, 3 (1), 121-132,(2010)

Salgado, F., J. Corrales, L. Muñoz, y J. Delgado, Diseño de programas de asignaturas basados en competencias y su aplicación en la Universidad del Bío-Bío, Chile, http://dx.doi.org/10.4067/S071833052012000200013, Ingeniare. Revista chilena de ingeniería, 20(2), 267-278, (2012)

Schuck, A.S. Gordon, y J. Buchanan, What are we missing here? Problematising wisdoms on teaching quality and professionalism in higher education,DOI:10.1080/13562510802334772,Teaching in Higher Education, 13(5), 537-547, (2008)

Soria-Barreto, K. y S. Zúñiga-Jara, Aspectos Determinantes del Éxito Académico de Estudiantes Universitarios. http://dx.doi.org/10.4067/S0718-50062014000500006, Formación universitaria, 7(5), 41-50, (2014)

Torres, E. y L. Araya, Construcción de una escala para medir la calidad del servicio de las universidades: Una Aplicación al Contexto Chileno, http://www.scielo.org.ve/scielo.php?pid=S131595182010000100006\&script=sci_arttext, ISSN 1315-9518, Revista de Ciencias Sociales v.16 n.1,(2010)

Tünnermann, C.,Calidad, evaluación institucional, acreditación y sistemas nacionales de acreditación,http://www.uam.ac.pa/pdf/tema_interes_1_evaluacion_acreditacion_uam.pdf,Instituto Latinoamericano de Educación para el Desarrollo,(2000)

UNESCO,Conferencia Mundial sobre la Educación Superioren el Siglo XXI: Visión y Acción, http://unesdoc.unesco.org/images/0011/001163/116345s.pdf, Tomo 1, Informe Final, (1998a)

UNESCO, Declaración Mundial sobre la Educación Superior. La Educación Superior en el Siglo XXI: Visión y Acción, disponible en: http://www.unesco.org/education/educprog/wche/declaration_spa.htm, (1998b)

Zhang, L.; Z. Han, y Q. Gao, Empirical study on the student satisfaction index in higher education.http://ccsenet.org/journal/index.php/ijbm/article/download/1256/1219, International Journal of Business and Management, 3 (9), 46-51, (2008) 